\title{
Explorando o paradigma Publish/Subscribe e a elasticidade em níveis aplicados ao procedimento de Telemedicina
}

\author{
Euclides Palma Paim ${ }^{1}$, Rodrigo da Rosa Righi ${ }^{1}$ and Cristiano André da \\ Costa $^{1}$
}

${ }^{1}$ Programa de Pós-Graduação em Computação Aplicada (PIPCA), Universidade do Vale do Rio dos Sinos (Unisinos), São Leopoldo (RS) - Brasil

*euclidespaim@gmail.com; rrrighi@unisinos.br; cac@unisinos.br

Submetido: 02/10/2017. Revisado: 23/03/2018. Aceito: 15/04/2018.

\begin{abstract}
Resumo
Imagens médicas são usadas diariamente para apoio ao diagnóstico em diferentes áreas da Radiologia no mundo todo. No entanto, há uma grande necessidade de que essas imagens sejam analisadas por diferentes especialistas e discutidas de forma ampla na busca do melhor tratamento para cada patologia. Em ambientes clínicos transmitir essas imagens em tempo hábil é um problema sem solução espontânea. A indisponibilidade de dados em tempo real para a avaliação médica especializada impacta diretamente no sucesso terapêutico. $\mathrm{O}$ modelo de computação em nuvem tem as características necessárias para garantir que estas imagens possam estar ao alcance de profissionais aptos a oferecer o melhor atendimento. 0 presente artigo apresenta o modelo PS2DICOM que estabelece comunicação em redes hospitalares e utiliza o paradigma Publicar/Assinar em dois níveis de escalabilidade. O modelo PS2DICOM é um middleware que atua na camada IaaS (Infrastructure as a Service), apoiando as tarefas de transmissão e armazenamento de arquivos dentro do padrão DICOM (Digital Imaging and Communications in Medicine). O sistema oferece ainda compactação dos dados de forma adaptativa à largura de banda disponível. A pesquisa contribui ao apresentar uma arquitetura eficaz para otimizar tarefas de rede, capaz de ser adotada como solução ao desenvolver aplicações voltadas para redes hospitalares. A arquitetura foi testada utilizando um protótipo com módulos distintos, desenvolvidos para cada serviço específico oferecido e mostrou-se eficiente como solução para os problemas em questão.
\end{abstract}

Palavras-Chave: Computação em nuvem; publicar/assinar; telemedicina.

\begin{abstract}
Medical images are used daily to support diagnosis in different areas of Radiology throughout the world. However, there is a great need for these images to be analyzed by different specialists and widely discussed in the search for the best treatment for each pathology. In clinical environments transmit these images in a timely manner is a problem without spontaneous solution. The unavailability of real-time data for specialized medical evaluation directly impacts therapeutic success. The cloud computing model has the characteristics necessary to ensure that these images can be within the reach of professionals able to offer the best service. This paper presents the PS2DICOM model that establishes communication in hospital networks and uses the Publish/Subscribe paradigm in two levels of scalability. The PS2DICOM model is a middleware that acts on the IaaS (Infrastructure as a Service) layer, supporting the tasks of transmitting and storing files within the DICOM (Digital Imaging and Communications in Medicine). The system also offers data compression adaptive to the available bandwidth. The research contributes to presenting an efficient architecture to optimize network tasks, capable of being adopted as a solution when developing applications aimed at hospital networks. The architecture was tested using a prototype with distinct modules, developed for each specific service offered and proved to be efficient as a solution to the problems in question.
\end{abstract}

Key words: Cloud Computing; publish/subscribe; telemedicine. 


\section{Introdução}

A Telemedicina constitui uma importante ferramenta para prestação de serviços médicos, é definida pela WHO (World Health Organization), como uma forma de prestação de serviços de saúde, onde a distância é um fator crítico e onde há uso de TIC (Tecnologias de Informação e Comunicação) por profissionais de saúde, para o intercâmbio de informações válidas para o diagnóstico, tratamento e prevenção de doenças, pesquisa, bem como para a educação continuada de prestadores de cuidados de saúde WHO (1998). Seu desenvolvimento, entretanto, é moroso devido a problemas relacionados à disponibilidade de recursos computacionais adequados e da capacidade de transmissão de dados sobre a infraestrutura de rede disponível hoje em dia Maldonado et al. (2016); Kaur and Wasson (2015). É comum a utilização de recursos de Telemedicina quando não há disponibilidade de um médico especialista, para realização de consultas remotas. Frequentemente existe a necessidade da transmissão de imagens pela Internet como forma de auxiliar o diagnóstico. Além da ausência de especialistas em algumas localidades, é importante salientar que em algumas regiões do país a infraestrutura de rede de computadores é insuficiente para transmissão de grande quantidade de dados.

Imagens médicas desempenham um papel crucial na Telemedicina e proporcionam a possibilidade de um diagnóstico mais preciso e com menores taxas de insucesso terapêutico. Estas imagens no entanto, consomem uma grande quantidade de recursos para serem armazenadas ou transmitidas. Verifica-se a necessidade de que tais imagens sejam analisadas por diferentes especialistas com o propósito de facilitar o diagnóstico Anuja and Jeyamala (2015). Porém, há poucas formas de compartilhamento desses arquivos atualmente descritas na norma DICOM ISO:12052 (2016). Além disso, essa troca deve acontecer em tempo real, a fim de que o melhor tratamento possa ser posto em prática dentro do menor tempo possível. Há um demanda variável por recursos computacionais utilizados para manter um sistema de armazenamento de imagens médicas instalado fisicamente. Esses servidores, chamados de PACS Picture Archiving and Communication Systems, carecem de recursos de comunicação entre redes distintas, onde hospitais distintos não possuem canais de comunicação entre seus serviços de imagens médicas Doel et al. (2017). Considerando o potencial escalar de geração de dados com que provedores de saúde tem que lidar e as características de elasticidade que o modelo de nuvem oferece para lidar com esses dados, uma solução natural seria adotar a nuvem para escalar a transmissão e o armazenamento, bem como os metadados relacionados Ojog and Arias-Estrada (2013); Teng et al. (2010).

\section{Referencial Teórico}

\subsection{Computação em Nuvem}

A computação em nuvem pode ser definida como um modelo que oferece acesso a determinados recursos alocados remotamente, sob demanda do cliente através da rede. Onde o provedor do serviço disponibiliza grupos de recursos computacionais que podem ser configurados a gosto pelo usuário Mell and Grance (2011); Puthal et al. (2015). Esses recursos podem ser caracterizados como o número de nós computacionais executando determinada tarefa, as configurações de redes e acesso remoto, número de processadores disponíveis em determinado nó, o espaço de armazenamento oferecido e bancos de dados, etc. Caracterizam esse modelo também, os aplicativos e serviços que podem ser alocados e liberados em tempo real quase sem necessidade de gerenciamento ou interação para configuração por parte do cliente como consta em Mell and Grance (2011); Puthal et al. (2015); Varia and Mathew (2014). O NIST (National Institute of Standards and Technology) define de forma bem clara as características principais relacionadas à concepção de computação em nuvem. Existem também, três modelos de serviço básico descritos na literatura (IaaS, SaaS, PaaS). Há ainda quatro formas de implantação da computação em nuvem definidas pelo instituto conforme os níveis de privacidade Mell and Grance (2011); Puthal et al. (2015).

\subsubsection{Características Principais}

O consumidor pode, por ele mesmo, requerer cargas de computação, como mais processadores ou espaço de armazenamento, conforme sua necessidade, através do navegador ou da SDK do provedor e sem requerer interação humana para alocar ou desalocar recursos por parte do provedor de serviços em nenhum momento. Os recursos de computação são agrupados para atender múltiplos consumidores ao mesmo tempo, compartilhando recursos de forma independente e individual. O consumidor utiliza os recursos de hardware e software de forma isolada sem conflito com os demais clientes e normalmente não tem controle sobre a localização exata dos recursos fornecidos. Todos os recursos fornecidos pelo provedor podem ser alocados ou liberados de maneira dinâmica, em alguns casos até mesmo de forma automática, mediante uma configuração prévia. Os sistemas em nuvem utilizam medidores de consumo de recursos, oferecendo métricas de medição para cada serviço utilizado, (por exemplo, número de processadores, número de unidades de armazenamento ou bancos de dados, utilização de banda e perfis de usuário). $O$ uso de recursos além de ser monitorado, controlado e relatado, proporciona ao cliente transparência ao permitir um controle sobre o que está sendo utilizado.

\subsubsection{Modelos de Serviços}

Existem três padrões de serviços ou modelos de implantação mais conhecidos para computação em nuvem como consta em Mell and Grance (2011), cada modelo adota características de acordo com o grau de controle que cada usuário tem em relação a infraestrutura. O cliente de cada modelo pode controlar apenas alguns aplicativos ou tomar decisões quanto a melhor plataforma operacional adotar. $\mathrm{O}$ cliente pode até mesmo exercer controle sobre a infraestrutura decidindo qual a melhor configuração de recursos adotar.

- Software como um Serviço (SaaS): Neste modelo 
de serviço é fornecido ao consumidor apenas recursos em forma de aplicativos do provedor com soluções em forma de softwares baseadas na nuvem. Essas aplicações podem ser acessadas de diferentes dispositivos. O serviço oferecido pode ser um aplicativo baseado na web por exemplo, onde o consumidor não tem controle sob a infraestrutura de nuvem subjacente diretamente, ficando esse a cargo do provedor. Contudo, o cliente pode realizar configurações específicas do aplicativo oferecidas pelo desenvolvedor Mell and Grance (2011); Puthal et al. (2015).

- Plataforma como um Serviço (PaaS): Neste modelo os recursos oferecidos ao consumidor são a infraestrutura necessária para criar aplicações em diferentes plataformas, usando linguagem de programação, bibliotecas, serviços e ferramentas suportados pelo provedor. Esta estrutura tem por fim, abstrair o desenvolvedor das preocupações com sistema, uma vez que a infraestrutura disponibilizada encontra-se um nível acima. $\mathrm{O}$ consumidor tem acesso apenas aos recursos da plataforma, não podendo controlar diretamente as configurações de hardware por exemplo, incluindo redes, servidores, sistemas operacionais ou armazenamento, mas pode escolher e controlar as aplicações a serem implantadas e possivelmente as configurações para esses aplicativos hospedados Mell and Grance (2011); Puthal et al. (2015).

- Infraestrutura como Serviço (IaaS): Nesta modalidade de serviço o consumidor tem 0 maior controle da configuração dos recursos computacionais a serem utilizados, como por exemplo número de processadores, capacidade de armazenamento, redes e demais recursos de computação importantes. Neste padrão de serviço o consumidor é capaz de lidar com diversos softwares e ainda instalar sistemas operacionais e aplicativos. O consumidor não tem controle sobre o hardware do provedor de nuvem diretamente, mas tem a liberdade de controlar o número de nós computacionais ativos e quais sistemas ou bancos de dados podem ser implantados. O cliente possui ainda algum controle limitado de componentes de rede distintos, podendo abrir portas TCP ou redirecionar tráfego através de Firewalls Mell and Grance (2011); Puthal et al. (2015).

\subsubsection{Modelos de Implantação}

Existem ainda formas de implantação, separando tipos de nuvens computacionais conforme suas características de privacidade em quatro grupos. Em nuvens privadas a infraestrutura da nuvem é qualificada para uso exclusivo por uma única instituição ou unidade de negócios, ficando sua utilização restrita a membros dessa organização, através de acesso identificado. A infraestrutura disponibilizada pode pertencer a organização que a gerencia, ou pode ser operada por um terceiro, podendo existir dentro ou fora das instalações da empresa. Nuvem comunitária neste formato de instalação a nuvem é criada para uso específico por uma comunidade de consumidores com objetivos em comum. Ela pode ser propriedade de organizações dessa comunidade ou terceirizada, podendo ainda possuir estrutura instalada fisicamente no local Mell and Grance (2011); Puthal et al. (2015).
Ao adotarmos nuvem pública estamos compartilhando a infraestrutura de um provedor, fornecida para uso comum pelo público em geral. Podendo ser propriedade de uma empresa, universidade ou organização específica. A infraestrutura encontra-se nas instalações do provedor de nuvem e está disponível para o uso coletivo. Nuvem híbrida: Neste modelo de implantação a nuvem adota uma forma combinada de duas ou mais infraestruturas de nuvem distintas (privadas, comunitárias ou públicas) que apesar de se manterem isoladas permanecem vinculadas por determinada tecnologia que permite a comunicação entre essas estruturas e o balanceamento de cargas de trabalho entre elas Mell and Grance (2011); Puthal et al. (2015).

Há uma demanda crescente por soluções em nuvem que supram as necessidades de clínicas e hospitais. Para facilitar a realização de tarefas complexas a computação em nuvem é uma arquitetura dominante, e pode eficientemente realizar cálculos de dados em larga escala Rallapalli et al. (2016). A fim de proporcionar maior qualidade no atendimento, diversos profissionais devem trocar informações sobre os pacientes. Esses dados, frequentemente são sigilosos e devem ser transmitidos de forma privada e segura. Devido a grande quantidade e ainda os diversos formatos, o ambiente de nuvem apresenta-se como a forma mais eficiente de armazenar e transmitir essa informação.

Dada a grande disponibilidade de recursos computacionais oferecidos pela computação em nuvem e o potencial disruptivo dessa arquitetura, a sua utilização é mais que uma tendência e torna-se uma necessidade para hospitais e clínicas atualmente. O impacto transformador do modelo de nuvem para o ambiente hospitalar pode ser compreendido a partir dos estudos apresentados no Capítulo 3. A adoção dessa infraestrutura para armazenamento, manipulação de dados e consulta em servidores PACS deve gerar economia de recursos e facilidades no gerenciamento de dados.

\subsection{Sistemas de Notificação de Eventos Distribuídos}

Um serviço de notificação de eventos distribuídos é um web service, que coordena e gerencia a entrega e o envio de mensagens, para end points registrados ou clientes. Existem dois tipos de clientes para esse modelo, os que publicam e os que assinam, também chamados de producers e consumers Teranishi et al. (2017); Coulouris et al. (2012). Clientes que publicam podem se comunicar rapidamente com assinantes através de mensagens enviadas a um tópico, que atua como canal lógico e assíncrono de comunicação. Essas mensagens podem ser trocadas utilizando protocolos conhecidos (HTTP/S, SMS, SMTP, etc.)

Estes sistemas são frequentemente adotados ao lidar com ambientes que possuem alta heterogeneidade, onde diferentes clientes comunicam-se a partir de plataformas ou ambientes de redes distintos. Podendo dessa forma conectar dispositivos que originalmente não foram projetados para trabalhar de forma conjunta Coulouris et al. (2012); Esposito et al. (2013). Além disso, podem ser 
usados para notificar mudanças em determinado objeto ou serviço em particular, a partir de assinaturas onde assinantes são notificados quando estas mudanças ocorrerem. Este desacoplamento de tempo e espaço entre objetos e clientes, promove diretamente a escalabilidade do sistema, uma vez que são eliminadas as dependências exigidas pelos clientes do modelo Eugster et al. (2003).

Sistemas baseados no paradigma Publicar/Assinar possuem normalmente três entidades fundamentais: um publicador, um assinante e um broker, ou serviço de notificação, responsável por interligar publicadores e assinantes. Os brokers permitem a comunicação entre os clientes do sistema através de tópicos, conteúdo, canal ou tipo de dados Coulouris et al. (2012); Tarkoma (2012). Também podem fornecer uma combinação dos mesmos, ou então como um recurso adicional baseado em filtragem, quando uma mensagem precisa ser entregue de modo específico a um assinante Tarkoma (2012).

o Modelo Publish/Subscribe é amplamente utilizado para lidar com eventos distribuídos em rede. Principalmente por suas características de comunicação assíncrona e pela facilidade de implementação e manutenção. Aplicado aos eventos relacionados ao fluxo de dados de TI clínicos padrão DICOM, esse modelo tem seu potencial amplamente explorado. Como sugere Westin et al. (2015), patente requerida para lidar com imagens médicas usando dispositivos móveis. Bem como as transformações exigidas ao manipular imagens dessa natureza descritas em Westin et al. (2016); Andersen et al. (2018).

\subsection{DICOM}

$\mathrm{Na}$ área pertencente ao campo de tecnologias da informática voltadas para a medicina, há normatizações internacionais para desenvolvimento de soluções e fabricação de aparelhos. Equipamentos para captura de imagens médicas e seus sistemas acessórios são amparados por uma dessas normatizações conhecida como DICOM ISO:12052 (2016). Para que dispositivos desenvolvidos para obtenção de imagens médicas possam funcionar com outros dispositivos de diferentes fabricantes foi estabelecido esse padrão internacional. Todo fabricante que reivindicar conformidade com a norma deve adotar as recomendações definidas neste compêndio Pianykh (2008); Maani et al. (2012).

Esta normatização dá ênfase às imagens médicas de diagnóstico, estabelecendo um arcabouço para disciplinas médicas que atuam com a obtenção de imagens e com o fluxo de dados de TI clínica. Esse recurso de apoio ao diagnóstico é utilizado na radiologia, cardiologia, odontologia, oftalmologia e disciplinas afins, bem como nas terapias baseadas em imagem como radiologia intervencionista, radioterapia e cirurgia. O padrão DICOM ISO:12052 (2016) é adotado como referência também para a medicina veterinária e seus ambientes médicos. Este padrão pode ser aplicado a um largo espectro de procedimentos de diagnose, bem como para os dados que acompanham esses estudos e para os exames relacionados.

Este padrão tem como principal finalidade, facilitar o relacionamento e a interoperabilidade entre sistemas que reivindicam conformidade à norma em questão. Surgiu da necessidade de estabelecer comunicação entre diferentes fabricantes de dispositivos de captura de imagem atuando no mercado Pianykh (2008). DICOM não é por si só uma garantia de interoperabilidade, cabendo a cada implantação adotar as medidas necessárias para o seu correto funcionamento. DICOM serve como referência internacional para serviços relacionados a imagens médicas e seus metadados, é parte do esforço conjunto entre a ACR (American College of Radiology) e da NEMA (National Electrical Manufactures Association) o padrão faz parte da ISO 12052:2006 Mildenberger et al. (2002).

O desenvolvimento do padrão DICOM ocorreu através da inciativa e cooperação entre a ACR e a NEMA, citadas anteriormente, que fundaram um comitê para estudar formas de comunicar dados entre fabricantes distintos. No ano de 1985 essas duas entidades publicaram o que pode ser chamado hoje de versão 1.0 da norma. Nos anos seguintes foram feitas revisões, e novas verões foram lançadas, até o ano de 1993, onde foi apresentada a versão 3.0 chamada de "Digital Communications in Medicine." A principal característica dessa versão foi a inclusão de um protocolo de rede baseado no modelo de referência OSI (Open System Interconnection) e o uso de TCP/IP como forma de garantir interoperabilidade entre diferentes desenvolvedores Mildenberger et al. (2002).

\section{Trabalhos Relacionados}

\subsection{Metodologia}

Como forma de encontrar os melhores resultados ao propor o modelo em questão, foram revisadas as soluções com características semelhantes ao sistema sugerido. Assim foi posta em ação uma estratégia para encontrar trabalhos relacionados à pesquisa, para que os mesmos pudessem ser estudados e inseridos neste artigo Fernández-Sáez et al. (2013). Nos artigos analisados, procurou-se identificar lacunas que o presente modelo possa preencher, bem como as técnicas que representem o estado da arte da disciplina, a fim de acrescentá-las a arquitetura final.

Para este artigo foi realizada uma revisão sistemática da literatura em trabalhos publicados nos últimos 5 anos, utilizando os seguintes termos: Publisher/Subscriber, DICOM, Compression, Healthcare, Medical Images, Cloud Computing. Foram utilizados os mecanismos de busca científicos mais conhecidos como Google Acadêmico ${ }^{1}$, PubMed ${ }^{2}$, Science Direct ${ }^{3}$, bem como o portal de periódicos CAPES/MEC 4. Buscou-se identificar os artigos relacionados com o tema que fossem publicados em periódicos revisados e conferências relacionadas, a seguir foram revistas as referências desses artigos para permitir uma compreensão mais abrangente do

\footnotetext{
1 https://scholar.google.com.br

${ }^{2}$ https://www.ncbi.nlm.nih.gov/pubmed/

3http://www.sciencedirect.com/

4http://www.periodicos.capes.gov.br/
} 
Tabela 1: Número de artigos identificados em cada fase da pesquisa

\begin{tabular}{lllllll}
\hline Termo Descritor & Fase I - Identificação & Fase II - Análise & Snowball & Fase -III & Snowball & Total \\
\hline Compression & 10 & 10 & 2 & 1 & 1 & 14 \\
Cloud Computing & 10 & 10 & 3 & 1 & 1 & 15 \\
Pub/Sub & 8 & 8 & 1 & 1 & 1 & 11 \\
Telemedicine & 3 & 3 & 5 & 1 & 1 & 10 \\
Healthcare & 3 & 3 & 1 & 2 & 1 & 7 \\
Total & 34 & 34 & 12 & 6 & 5 & 57 \\
\hline
\end{tabular}

assunto e um melhor embasamento teórico.

Os artigos adquiridos em cada fase da pesquisa (descritos na Tabela 1) foram anexados a biblioteca do aplicativo Google Acadêmico a fim de facilitar a manipulação e leitura dos mesmos. Foi adotado um critério de exclusão dos artigos publicados em mais de uma base de pesquisa, artigos cujo os termos de pesquisa tenham apenas sido mencionados no resumo do trabalho também foram excluídos. Adicionalmente, foi aplicado um critério de discernimento em relação aos trabalhos mais atuais e relevantes a serem apresentados no presente artigo.

A busca por trabalhos pode ser separada em três fases principais como sugerem FernándezSáez et al. (2013), na fase inicial foram revisadas as referências literárias mais relevantes ao tema utilizando os termos destacados. A partir dos resultados obtidos nos motores de busca, foram selecionados os trabalhos mais importantes de acordo com os critérios descritos acima. Na fase seguinte, todos os trabalhos selecionados foram lidos. Com base nas informações obtidas, inicia-se a terceira fase onde uma nova busca é realizada e novas referências são inseridas. O processo então é reiniciado e repetido até que todo o tema ou a sua parte mais importante possa ser completamente revisada. Os resultados obtidos, sobretudo depois de repetido o procedimento algumas vezes, foram então submetidos a uma triagem e os artigos selecionados compõem a presente seleção.

\subsection{A Large-Scale Data Collection Scheme for Distributed Topic-Based Pub/Sub}

Neste artigo os autores propõem um esquema de encaminhamento e armazenamento de mensagens em uma rede overlay para IoT. O trabalho assemelhase ao modelo desenvolvido, pois aborda aspectos da comunicação no modelo Pub/Sub entre diferentes dispositivos, utilizando a nuvem como uma rede de sobreposição Teranishi et al. (2017).

Os autores argumentam que é possível reduzir o processamento das cargas computacionais mesmo com um grande número de mensagens trafegando pela rede, mantendo o tempo de entrega controlado durante a comunicação. Para isso foi usado um método adaptativo de coleta de mensagens, distribuindo a carga entre entre brokers de rede, evitando sobrecargas no sistema. Cada broker contém um publicador ou um assinante, quando um dispositivo tenta enviar ou receber mensagens para um tópico, o mesmo cria uma rede de sobreposição para a realização da tarefa de forma distribuída.

$\mathrm{O}$ método proposto agrupa mensagens de mesmo tamanho a fim de serem enviadas em pedaços agrupados, ao invés do envio de pequenos segmentos.

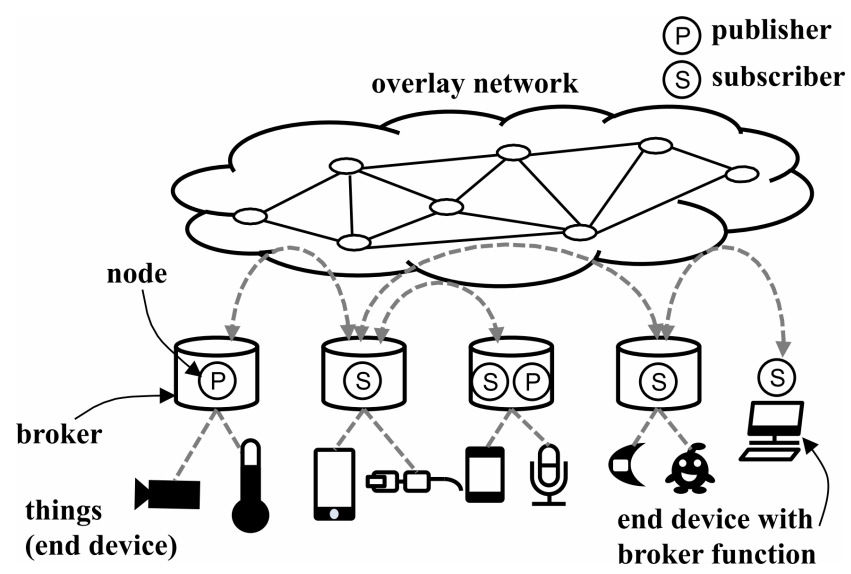

Figura 1: Visão geral da arquitetura sugerida pelo autor Teranishi et al. (2017)

Os resultados foram obtidos utilizando um protótipo baseado em máquinas virtuais em nuvem e um framework Java. Os autores afirmam que o tempo de ocupação da rede foi reduzido em aproximadamente $90 \%$. Contudo, o trabalho em questão não aborda soluções relativas ao armazenamento ou backup dessas mensagens, tampouco sugere níveis de balanceamento das cargas de trabalho atuantes no sistema.

\subsection{Systems and Devices for Encrypting, Converting and Interacting with Medical Images Using a Mobile Device}

Este trabalho refere-se ao pedido de registro de patente "US-20150278444-A1", e diz respeito a um método de encriptação, conversão e transferência de arquivos no formato de imagens médicas para dispositivos móveis Westin et al. (2015). O presente registro descreve um sistema e equipamentos necessários para tratar da comunicação de imagens médicas e metadados, entre dispositivos que reivindicam conformidade com o padrão DICOM e aparelhos móveis conectados em redes sem fio.

Os autores registraram ainda um método para conversão das imagens para formatos compatíveis com as plataformas de destino. Tais arquivos podem, além de serem visualizados em diferentes plataformas móveis, receberem anotações de vários especialistas envolvidos no estudo e serem reenviadas para o servidor. O registro contudo não aborda a questões como compactação sem perdas dessas imagens, deixando de considerar o tamanho dos arquivos bem como o tempo de comunicação entre publicador e assinante. 


\subsection{Healthcare Systems Integration Using Real Time Publish Subscribe (RTPS) Middleware}

Neste artigo os autores desenvolvem e testam um middleware, baseado no paradigma publicar/assinar, para lidar com sistemas da área da saúde. Segundo os autores, a questão mais desafiadora ao implementar tais sistemas é lidar em tempo real, com a heterogeneidade associada a diferentes dispositivos presentes na rede Almadani et al. (2016). Essa miríade de equipamentos pode causar atrasos na comunicação das mensagens, o que seria diretamente impactante na condição de saúde dos pacientes. Para tanto, a abordagem utilizando um modelo que integre em tempo real diferentes sistemas de forma assíncrona, torna-se uma necessidade para essa área da Telemedicina.

Desta forma conforme sugerem os autores, o middleware apresentado é capaz de desacoplar espacialmente diferentes nós da rede envolvidos no processo. Os dados relacionados podem ser entregues imediatamente após a sua publicação ou sofrerem atrasos conforme demanda. E o fluxo de informação na rede pode ser controlado de acordo com a largura de banda disponível, para isso o middleware fornece um conjunto de APIs capaz de lidar com diferentes perfis de uso da aplicação. Nessa solução, mais uma vez, os desenvolvedores não consideram o tamanho das mensagens trafegando no sistema, não apresentando nenhum tipo de compressão para lidar com o conjunto de dados durante a tarefa de comunicação.

\subsection{ROI Based Medical Image Compression for Telemedicine Application}

Imagens médicas possuem três partes distintas, conhecidas como ROI Region of Interest, não ROI, e background, sendo a primeira a região mais crítica dentro de uma imagem, como demonstra a Figura 2. Algumas técnicas de compressão encaram as imagens por regiões de interesse, descartando áreas sem importância para o diagnóstico, diminuindo o tamanho final do arquivo Kaur and Wasson (2015). Neste trabalho os autores apresentam uma técnica híbrida de compressão sem perdas, onde são aplicadas diferentes tipos de compactação em regiões distintas da imagem.

A solução sugerida aqui aplica compressão com pequenas perdas de qualidade lossy data compression a regiões de não interesse e background. Ao passo que, nas regiões de interesse da imagem é aplicada compressão sem perdas lossless, com base na leitura dos pixeis do arquivo. Por fim, a imagem é então reagrupada, obtendo uma compressão híbrida, sem comprometer a qualidade dos dados em estudo. De acordo com os autores, as técnicas apresentadas geram resultados mais precisos e rápidos na aplicação da compressão, comparados aos estudos anteriores relacionados neste caso.

\section{Modelo PS2DICOM}

O modelo PS2DICOM trata imagens geradas por dispositivos médicos provenientes de diferentes instituições, dentro um padrão específico, e

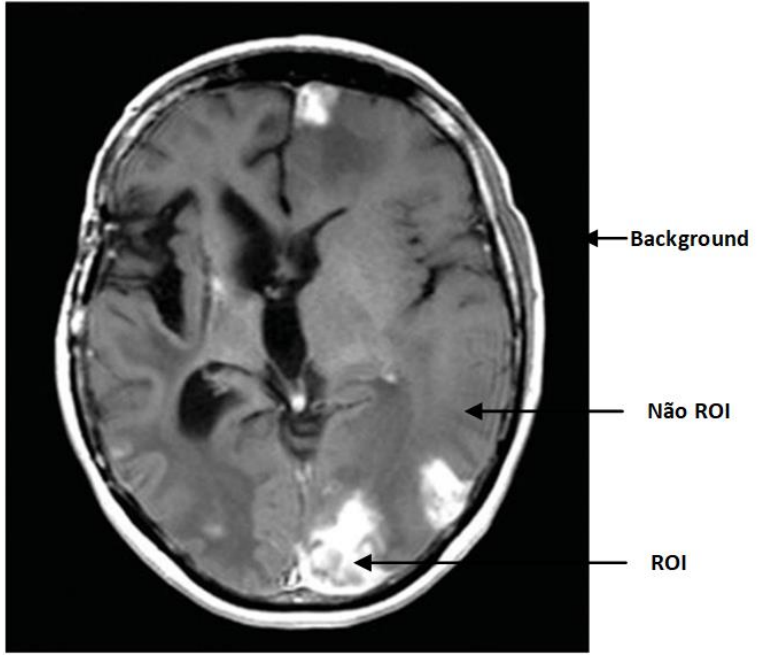

Figura 2: Imagem padrão DICOM mostrando diferentes regiões de interesse

disponibiliza estes arquivos entre especialistas em interpretação e diagnósticos. A arquitetura proposta viabiliza o armazenamento seletivo e a transmissão dessas imagens baseado em eventos no paradigma Publish/Subscribe Esposito et al. (2014). Cada hospital membro de uma rede de clínicas atua como um nó, compartilhando dados de forma particular com os demais parceiros. Estes dados são armazenados na nuvem e são retransmitidos conforme solicitações ocorrem no sistema. Os brokers atuam de forma dinâmica, convertendo os dados em formatos mais populares quando necessário e propagando as informações em tópicos entre membros da rede.

$O$ modelo oferece elasticidade horizontal e reativa em dois níveis, com base em variáveis do sistema, a Figura 3 apresenta uma visão de alto nível do modelo PS2DICOM. Dessa maneira os recursos podem ser adaptados a demanda da aplicação e ao consumo de recursos computacionais em tempo real. Tal estratégia proporciona economia na utilização da computação em nuvem, uma vez que é aprovisionada apenas a infraestrutura necessária para o correto funcionamento do serviço. PS2DICOM pode comprimir arquivos de forma adaptativa antes de sua transmissão, com base em diferentes métricas, como sugere Ootsu et al. (2016). Através de um recurso capaz de avaliar a eficiência da rede no momento da comunicação dos dados, o sistema pode optar por aplicar menos ou mais compressão no conjunto de arquivos.

\subsection{Arquitetura}

O modelo PS2DICOM concentra-se na integração de dados originados em diversas fontes relacionadas a saúde, operando no nível IaaS. É uma arquitetura em nuvem capaz de disponibilizar o compartilhamento de arquivos de imagens e metadados (informações do paciente) no padrão DICOM e obter vantagem da elasticidade em nuvem. Podemos inferir que esta arquitetura é capaz de facilitar a obtenção de informações importantes sobre o estado de saúde do paciente, uma vez que ela é capaz de integrar diversos especialistas em diagnose, através 


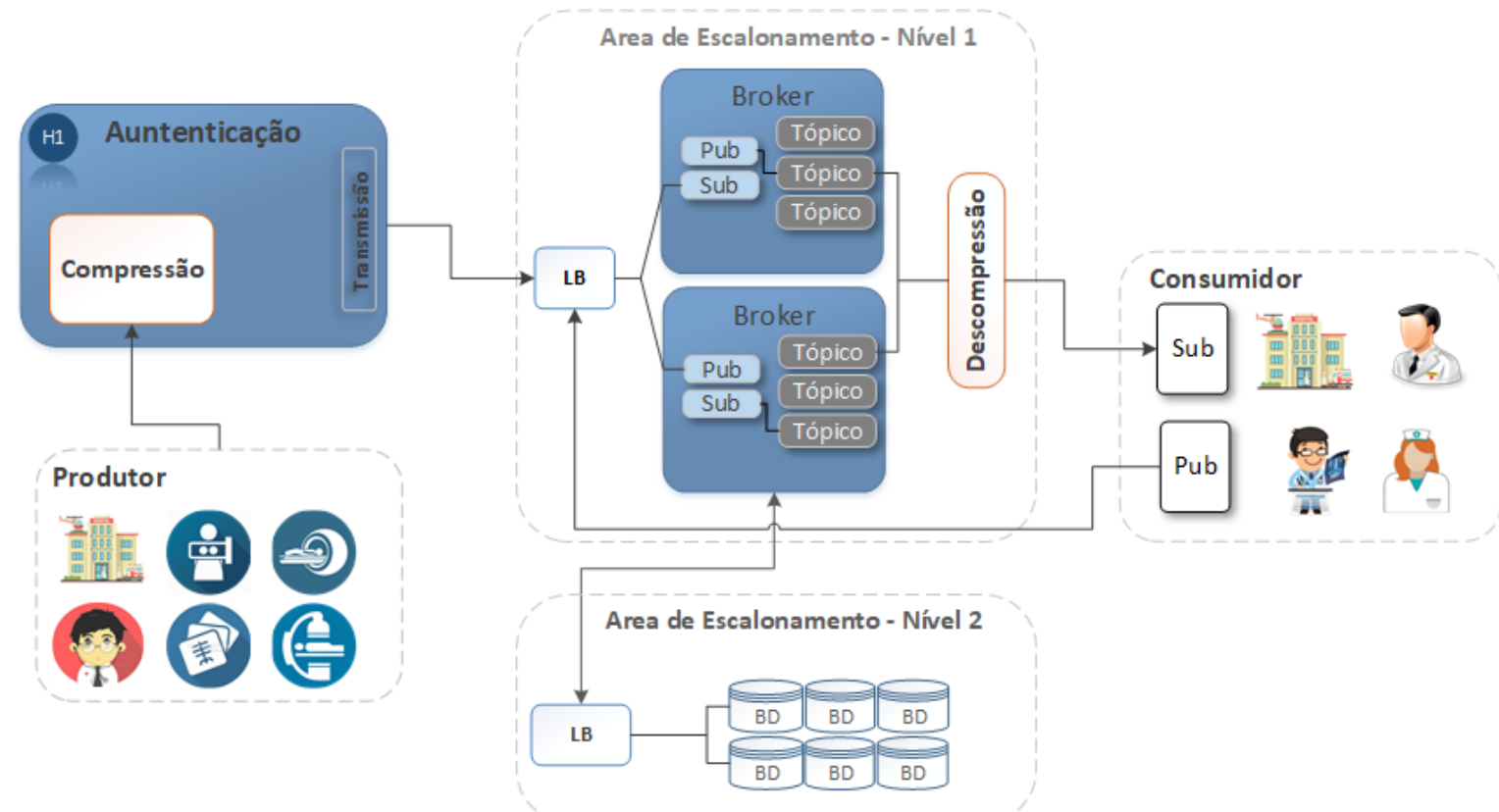

Figura 3: Visão de alto nível apresentando as características fundamentais do modelo PS2DICOM

da troca de mensagens modeladas pelo paradigma $\mathrm{Pub} / \mathrm{Sub}$. Arquitetado para extrair as vantagens desta infraestrutura o modelo PS2DICOM viabiliza acesso e armazenamento de forma escalar e elástica. Através Figura 3 podemos ver as características presentes na arquitetura em questão.

O modelo possui um módulo de comunicação de dados localizado nos hospitais ou clínicas da rede, capaz de comprimir os arquivos antes de sua transmissão, com base na qualidade da comunicação no momento do envio e tamanho do conjunto de dados, como sugerem Ootsu et al. (2016). Estas funcionalidades estão ilustradas na Figura 3. Além de comprimir, este módulo tem a capacidade de autenticar requisições de diferentes clientes.

A nuvem possui balanceamento de carga em dois níveis, de forma a distribuir as requisições entre diferentes instâncias e reter apenas os recursos computacionais exigidos pelas cargas de trabalho naquele momento, comportando-se de forma elástica. No primeiro estágio, descrito como Level 1 na Figura 3, temos um load balancer que gerencia e distribui as solicitações entre diferentes brokers. Estas máquinas virtuais são responsáveis pela retransmissão dos dados requisitados entre os assinantes de cada tópico. Os inscritos devem manifestar interesse em determinado assunto como consta em Coulouris et al. (2012). Uma vez confirmada a assinatura, os brokers passam a direcionar as mensagens enviadas por publicadores para os inscritos desse tópico.

No segundo nível, descrito como Level 2 na Figura 3, encontramos outro load balancer capaz de alocar de forma elástica as necessidades do modelo entre instâncias de bancos de dados. Neste nível são tratadas requisições provenientes de diferentes brokers, o que aumenta a disponibilidade dos recursos computacionais neste estágio. PS2DICOM pode garantir acesso aos dados armazenados, uma vez que diferentes operações de leitura e escrita podem acorrer de forma simultânea, podendo ser escaladas conforme demanda, promovendo paralelismo na comunicação com as instâncias deste estágio.

O modelo foi desenvolvido em módulos que facilitam a implementação de suas interfaces, conforme sugerem Coulouris et al. (2012); Bastiao Silva et al. (2012). Para estabelecer comunicação em tempo real entre diferentes recursos locais e a nuvem, o modelo PS2DICOM segue o paradigma da comunicação para sistemas distribuídos Pub/Sub. É ainda levada em consideração a qualidade da rede e a quantidade dados no momento da transmissão a fim de se adotar a melhor técnica de compressão adaptativa, introduzida como importante recurso para melhorar a eficiência da tarefa de comunicação.

\subsection{Autenticação e Avaliação da Capacidade de Transmissão}

A fim de tornar mais eficiente a comunicação entre diferentes redes hospitalares, o modelo PS2DICOM implementa este recurso. Esta componente atua como um serviço capaz de autenticar usuários do sistema e avaliar a qualidade da rede no momento da transmissão. Este serviço também é responsável por definir qual a melhor taxa de compactação a ser aplicada ao conjunto de dados. Localizado no lado do cliente este módulo visa diminuir o tempo de comunicação entre os hospitais e a nuvem. Este recurso utiliza o algoritmo (Gzip ${ }^{1}$ ), e pode aplicar diferentes níveis de compressão de acordo com o tipo de dado a ser transmitido. Com base na qualidade da rede e no total de arquivos a serem enviados, este módulo será capaz de decidir a melhor estratégia de compressão a ser adotada.

Uma vez conectado ao sistema, o modelo realiza testes automáticos na rede e aguarda a seleção

${ }^{1}$ http://www.gzip.org/ 
dos arquivos a serem transmitidos. De posse da velocidade de upload obtidas no teste inicial e considerando o volume de dados selecionados, o modelo PS2DICOM define se é necessário aplicar compressão e qual a taxa ideal a ser usada. Pode inclusive, enviar mensagens sem realizar compactação ou ainda aplicar o grau máximo de compressão caso a soma dos arquivos ultrapasse o limite pré definido pelo usuário. Compactando diferentes quantidades de dados de forma distinta e utilizando níveis de compressão adequados a cada caso, esse componente pode incrementar a eficiência na comunicação entre entidades. Os algoritmos utilizados para compressão derivam do LZ77, desenvolvido por Abraham Lempel e Jacob Ziv em 1977. São capazes de aplicar compressão sem perdas (lossless) ao conjunto de dados. Foram selecionados a partir de testes desenvolvidos para identificar os melhores algoritmos para compressão e descompressão das imagens padrão DICOM e metadados incluídos.

\subsection{Controlador de Notificações Pub/Sub}

Capaz de lidar com a demanda de imagens proveniente de servidores localizados em diferentes hospitais esse módulo é responsável por receber arquivos e encaminhar para seus destinatários. Localizado no lado do provedor, esta interface pode estabelecer comunicação de um para um ou um para muitos clientes. Com base em tópicos de interesse, este componente implementa o conceito publicar/assinar e estabelece um canal de comunicação em tempo real entre clientes do modelo PS2DICOM. Ao utilizar mensagens dentro do padrão pub/sub para trafegar as informações do modelo, esse modulo é capaz de melhorar a eficiência da tarefa de comunicação de dados dentro do padrão DICOM, consolidando a integração entre diferentes especialistas.

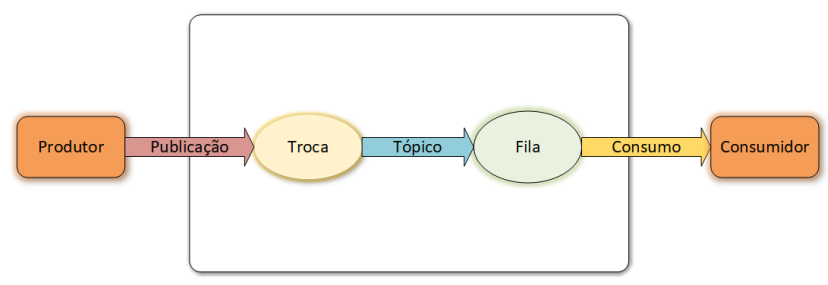

Figura 4: Diagrama representando o paradigma publisher/subscriber

Esse serviço está apto a colher as mensagens provenientes de diferentes hospitais ou clínicas da rede, e encaminhar as requisições aos seus respectivos destinatários. Uma cópia então é criada e armazenada em instâncias seguras implantadas na nuvem, para backup e futuras consultas. Este módulo é responsável por gerenciar os fluxos de dados entre entidades da rede e fornecer balanceamento de carga para brokers quando for necessário. A Figura 4 ilustra a dinâmica do fluxo de mensagens no modelo em estudo.

\subsection{Elasticidade Level 1 Brokers}

Elasticidade é um recurso chave ao implantarmos computação em nuvem e está entre as características do modelo que devem ser oferecidas pelo middleware adotado. Quando utilizamos computação em nuvem podemos fazer uso de rotinas definidas ao lidar com as cargas de trabalho que ultrapassam os recursos alocados previamente, sem comprometer desempenho e funcionalidades do sistema. Analisar os recursos disponíveis e disponibilizá-los de acordo com a carga de serviço vai garantir uma economia no custo final da operação Righi (2013). A abordagem reativa automática é tradicional e está disponível na maioria dos middlewares de nuvem comerciais disponíveis atualmente. Parte fundamental do modelo PS2DICOM, a elasticidade oferecida no primeiro nível da arquitetura visa garantir variabilidade dos recursos disponíveis conforme demanda.

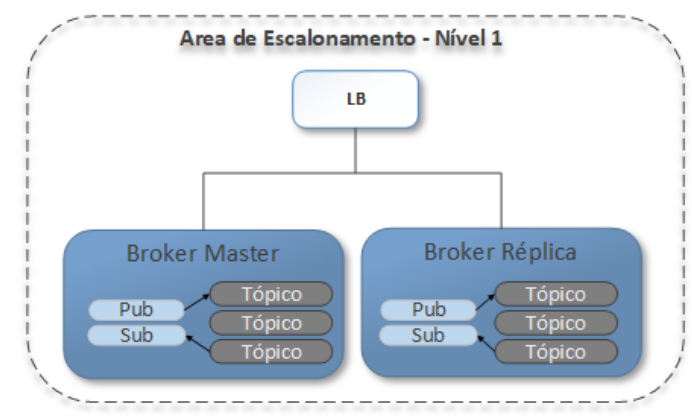

Figura 5: Componentes do primeiro nível de escalonamento

Neste módulo da arquitetura, as requisições provenientes de diferentes instituições conectadas ao sistema são recebidas em um ponto único, como mostra a Figura 5, o qual é responsável por aumentar ou diminuir a quantidade de brokers ativos. Com base em thresholds de utilização de CPU, esse load balancer é capaz de concentrar as demandas provenientes dos clientes, e replicar brokers caso a taxa de ocupação ultrapasse um desses limites. Este balanceador garante estabilidade ao modelo quando houver picos de utilização ou ainda desligando instâncias inativas, quando as mesmas deixarem de ser necessárias por um certo período de tempo.

Com base na solução de elasticidade apresentada em Righi et al. (2016), o modelo atual é capaz de alocar recursos, no primeiro nível de elasticidade, sem a necessidade de intervenção do usuário. Ao monitorar periodicamente a carga de utilização da CPU, essa solução é capaz de alocar novas máquinas virtuais para executar processos escravos que realizam requisições ao load balancer em portas de rede diferentes (listeners). Essa ação é controlada pelo balanceador de carga e somente após sua inicialização completa a nova VM passa a responder requisições provenientes do sistema.

O modelo PS2DICOM pode finalizar de forma automática a utilização de uma VM a partir do monitoramento do uso de CPU. Uma vez que a taxa de ocupação deste recurso atinja o threshold mínimo 
definido durante determinado intervalo de tempo, o balanceador de carga encarrega-se de encaminhar as requisições dos processos escravos para a última VM inicializada e desativa a VM subutilizada, mantendo pelo menos um broker em funcionamento para responder as demandas dos sistema. Essa ação de elasticidade diminui o consumo por recursos computacionais do middleware de nuvem em uso.

\subsection{Elasticidade Level 2 Bancos de Dados}

No estágio seguinte da arquitetura apresentada, temos outro balanceador de carga, este responsável por receber as requisições internas de diferentes brokers e distribuí-las entre instâncias de banco de dados, dedicadas ao armazenamento das imagens em estudo. A fim de garantir que os dados tratados pelo modelo estejam disponíveis para futuras consultas, essa técnica de armazenamento permite que imagens já arquivadas possam ser consultadas a partir da nuvem, sem a necessidade de um novo upload Ao concentrar os arquivos DICOM em instâncias na nuvem, estamos melhorando a eficiência na comunicação entre publicadores e assinantes.

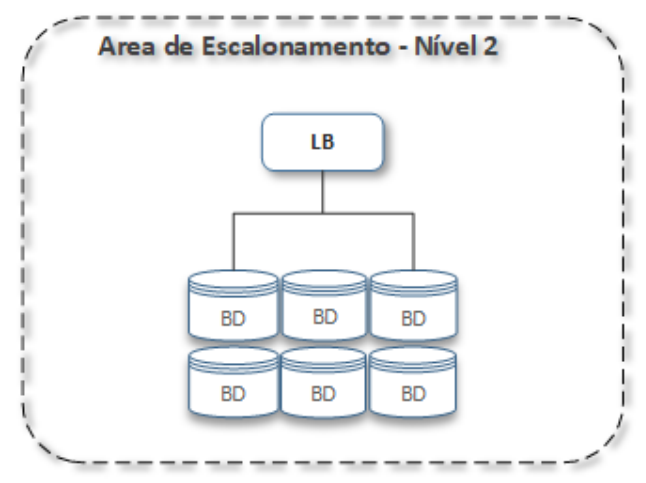

Figura 6: Componentes do segundo nível de escalonamento

Neste estágio, o modelo PS2DICOM implementa ações de elasticidade com base limites de utilização de hardware, dessa forma novas VMs dedicadas ao armazenamento de imagens podem ser alocadas ao serem atingidos thresholds pré definidos, sem necessidade de intervenção por parte dos usuários do sistema. O modelo aqui foi adaptado para disparar novas VMs de bancos de dados a partir da taxa de ocupação de disco. Ou seja, quando recursos de armazenamento ultrapassam os limites definidos de antemão, o load balancer instancia nova VM e redireciona as requisições de escrita provenientes do sistema através de uma porta de rede diferente (listener).

\section{Resultados}

Esta Seção descreve os resultados obtidos em todos os testes realizados, nos diferentes ambientes e cenários definidos. Os resultados associados a métrica Mensagens por Segundo são apresentados na Subseção 5.1. A Subseção 5.2 apresenta os tempos obtidos durante a execução do algoritmo de compressão e upload, bem como os tempos de download e descompressão dos arquivos DICOM. A análise da métrica Nível de Compressão é feita na Subseção 5.3.

\subsection{Análise das Métricas: Mensagens por Segundo}

A Figura 7 descreve o número de mensagens por segundo (throughput) publicadas e consumidas em um cenário de 1 produtor e 1 consumidor respectivamente, com uma carga de trabalho de 100.000 mensagens de $1 \mathrm{~KB}$ cada. Podemos observar que o broker transmite cerca de 3500 mensagens/s com esse workload.

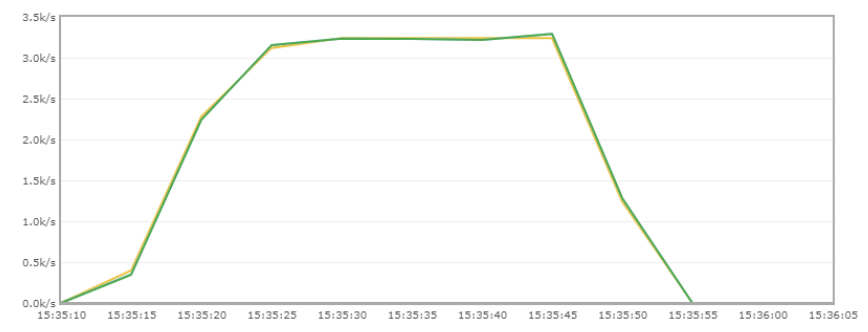

Figura 7: Número de mensagens/s em tamanho padrão produzidas e consumidas

A Figura 8 refere-se as mensagens publicadas e consumidas em um cenário de 1 produtor e 1 consumidor como no exemplo anterior. Nesta etapa a carga de trabalho foi aumentada e contém o equivalente a 10 imagens padrão DICOM por transmissão. Podemos observar que o broker transmite cerca de 75 mensagens/s com esse workload.

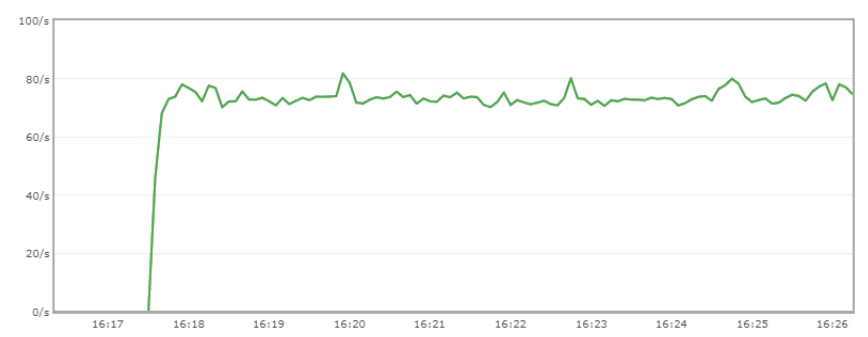

Figura 8: Número de mensagens/s com tamanho modificado produzidas e consumidas

\subsection{Análise de Desempenho: Tempo de Execução}

As Tabelas 2 e 3 apresentam o tempo de upload e download de imagens DICOM utilizando diferentes níveis de compactação aplicados ao conjunto de dados, comparados ao tempo de envio e recebimento dos mesmos dados sem o uso de compressão. Podemos observar que após compactadas as mesmas puderam ser transmitidas em menor tempo, ainda que utilizando graus mais intensos de compactação. Este comportamento sugere que a compressão de 
Tabela 2: Tempo de upload utilizando diferentes graus de compressão

\begin{tabular}{|c|c|c|c|c|c|c|}
\hline Dados DICOM & ZIP Level 0 + Up & UnZip Level 0 + Down & ZIP Level $2+$ Up & UnZip Level 2 + Down & ZIP Level 3 + Up & UnZip Level 3 + Down \\
\hline $10 \mathrm{MB}$ & $60,283 \mathrm{~s}$ & $6,751 \mathrm{~s}$ & 22,578 s & $5,347 s$ & $21,227 s$ & $5,572 \mathrm{~s}$ \\
\hline $50 \mathrm{MB}$ & $91,239 \mathrm{~s}$ & $16,602 \mathrm{~s}$ & $37,714 s$ & $11,118 \mathrm{~s}$ & $37,27 s$ & $9,33 \mathrm{~s}$ \\
\hline $100 \mathrm{MB}$ & $187,617 \mathrm{~s}$ & $42,346 \mathrm{~s}$ & $67,592 \mathrm{~s}$ & $19,602 \mathrm{~s}$ & $65,067 \mathrm{~s}$ & $15,587 \mathrm{~s}$ \\
\hline $200 \mathrm{MB}$ & $341,633 s$ & $72,951 \mathrm{~s}$ & $131,652 \mathrm{~s}$ & $29,491 s$ & $134,053 \mathrm{~s}$ & 28,189 s \\
\hline $400 \mathrm{MB}$ & $723,007 s$ & $132,251 \mathrm{~s}$ & $330,265 s$ & $74,396 s$ & $322,468 s$ & $79,015 s$ \\
\hline
\end{tabular}

Tabela 3: Tempo de upload utilizando diferentes graus de compressão

\begin{tabular}{ccccccc} 
Dados DICOM & ZIP Level 5 + Up & UnZip Level 5 + Down & ZIP Level 7 + Up & UnZip Level 7 + Down & ZIP Level 9 + Up & UnZip Level 9 + Down \\
\hline $10 \mathrm{MB}$ & $21,478 \mathrm{~s}$ & $5,590 \mathrm{~s}$ & $21,608 \mathrm{~s}$ & $6,067 \mathrm{~s}$ & $29,241 \mathrm{~s}$ \\
\hline $50 \mathrm{MB}$ & $38,627 \mathrm{~s}$ & $12,892 \mathrm{~s}$ & $41,643 \mathrm{~s}$ & $12,135 \mathrm{~s}$ & $61,774 \mathrm{~s}$ \\
\hline $100 \mathrm{MB}$ & $67,686 \mathrm{~s}$ & $17,067 \mathrm{~s}$ & $74,055 \mathrm{~s}$ & $19,760 \mathrm{~s}$ & $114,322 \mathrm{~s}$ \\
\hline $200 \mathrm{MB}$ & $138,142 \mathrm{~s}$ & $33,021 \mathrm{~s}$ & $148,053 \mathrm{~s}$ & $22,931 \mathrm{~s}$ & $226,310 \mathrm{~s}$ & $17,002 \mathrm{~s}$ \\
\hline $400 \mathrm{MB}$ & $333,034 \mathrm{~s}$ & $48,693 \mathrm{~s}$ & $355,713 \mathrm{~s}$ & $79,840 \mathrm{~s}$ & $380,094 \mathrm{~s}$ \\
\hline
\end{tabular}

arquivos DICOM antes do envio aumenta a eficiência da tarefa de transmissão, tornando o tempo de upload e download menores.

Os resultados obtidos neste teste sugerem que a aplicação de compressão nos dados DICOM colaboram com a melhoria da eficiência da tarefa de transmissão de arquivos em redes hospitalares. Uma vez compactadas essas mensagens podem ser enviadas e recebidas em menor tempo, mesmo considerando o tempo despendido para comprimir e descomprimir os arquivos antes do envio e depois do recebimento respectivamente.

\subsection{Análise das Métricas: Nível de Compressão}

A Figura 9 apresenta o tempo empregado para a execução de cada carga de trabalho utilizando diferentes algoritmos de compressão aplicados às imagens DICOM. O eixo X corresponde a quantidade de dados medidos em MegaBytes utilizados para cada teste. $\mathrm{O}$ eixo $\mathrm{Y}$ refere-se ao tempo medido em segundos e cada linha do gráfico representa o algoritmo empregado na compactação dos dados.

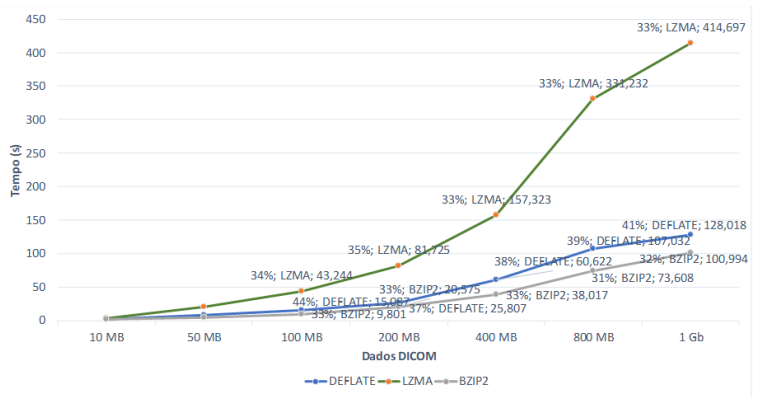

Figura 9: Tempo obtido aplicando diferentes algoritmos de compressão as imagens DICOM

Podemos perceber que o algoritmo DEFLATE apresentou os melhores resultados, compactando conjuntos de imagens DICOM de diferentes tamanhos em menor tempo comparado aos demais. Os resultados obtidos utilizando o algoritmo LZMA
(Lempel-Ziv Markov) demonstrou-se menos eficiente confrontado aos outros.

\section{Conclusão}

O grande número de imagens médicas geradas diariamente traz diversos desafios e oportunidades para a correta utilização desses dados em benefício do paciente. Usufruir dos recursos de infraestrutura oferecidos pela computação em nuvem para alavancar um modelo de compartilhamento e colaboração no diagnóstico baseado em imagens é o objetivo principal do modelo PS2DICOM. Neste trabalho, foram apresentados módulos que integram o fluxo de imagens gerados em ambiente hospitalar, a uma arquitetura desenvolvida em nuvem, para ampliar a eficiência na comunicação de informações nos modelos conhecidos de transmissão de dados dentro do padrão DICOM.

Conforme pesquisa no Tabnet ${ }^{2}$ (ferramenta de tabulação de dados de procedimentos do Sistema Único de Saúde), no mês de Julho de 2017, somente no Estado do Rio Grande do Sul foram realizados 10.046 exames de tomografia computadorizada de crânio. $\mathrm{O}$ número expressivo, evidencia o altíssimo quantitativo de procedimentos de diagnóstico apoiados em imagens, e a imensa importância que esses exames trazem para o processo de prevenção e tratamento do paciente.

Ao disponibilizarmos esses dados em tempo real a uma gama de profissionais, possibilitamos que um estudo terapêutico conjunto minimize exposição a radiações desnecessárias. Os dados ainda podem fomentar discussões sobre linhas de cuidado, apontar indicadores de saúde que podem proporcionar o mapeamento de áreas onde hajam pacientes com patologias semelhantes ou afins, e principalmente: assistir rapidez no atendimento, desoneração de custos de armazenamento e satisfação do usuário.

Foi apresentado um modelo que identifique a melhor forma de transmitir, armazenar, consultar e recuperar imagens DICOM em servidores PACS instalados em nuvens computacionais desenvolvidas com essa finalidade. A metodologia utilizada para

\footnotetext{
$2 \overline{\text { www.tabnet.datasus.gov.br/ }}$
} 
implementação desse ambiente de testes foi definida com base em um conjunto de métricas intrínsecas ao projeto, selecionadas a partir de uma análise de sensibilidade e das necessidades mínimas para um funcionamento correto da aplicação em questão.

Considerando os trabalhos estudados para composição deste artigo podemos salientar que o modelo PS2DICOM reúne um conjunto de soluções diferenciadas em relação ao estado da arte do tema em questão. A combinação do arquétipo publicar/assinar com a infraestrutura de recursos oferecidos em nuvem promove as condições ideais para a comunicação de imagens médicas em redes hospitalares. Não obstante, as técnicas de compressão adaptativa somadas aos demais recursos mencionados, inovam e ampliam a eficiência do procedimento de telemedicina.

\section{Agradecimentos}

Os autores gostariam de agradecer aos seguintes órgãos de fomento a pesquisa: CNPq, FAPERGS e CAPES.

\section{Referências}

Almadani, B., Saeed, B. and Alroubaiy, A. (2016). Healthcare systems integration using real time publish subscribe (rtps) middleware, Computers \& Electrical Engineering 50: 67-78.

Andersen, B., Kasparick, M., Ulrich, H., Franke, S., Schlamelcher, J., Rockstroh, M. and Ingenerf, J. (2018). Connecting the clinical it infrastructure to a service-oriented architecture of medical devices, Biomedical Engineering/Biomedizinische Technik 63(1): 57-68.

Anuja, M. and Jeyamala, C. (2015). A survey on security issues and solutions for storage and exchange of medical images in cloud, International Journal of Emerging Trends in Electrical and Electronics 11: 27-32.

Bastiao Silva, L. A., Costa, C. and Oliveira, J. L. (2012). A pacs archive architecture supported on cloud services, International Journal of Computer Assisted Radiology and Surgery 7(3): 349-358.

Coulouris, G., Dollimore, J. and Kindberg, T. (2012). Distributed Systems: Concepts and Design, Vol. 4. Disponível em http://www.amazon.com/dp/ 0321263545 (Accessado 2 Setembro 2017).

Doel, T., Shakir, D. I., Pratt, R., Aertsen, M., Moggridge, J., Bellon, E., David, A. L., Deprest, J., Vercauteren, T. and Ourselin, S. (2017). Giftcloud: A data sharing and collaboration platform for medical imaging research, Computer Methods and Programs in Biomedicine 139: 181-190. Disponível em http://dx.doi.org/10.1016/j.cmpb.2016.11.004 (Accessado 2 Setembro 2017).

Esposito, C., Ciampi, M. and De Pietro, G. (2014). An event-based notification approach for the delivery of patient medical information, Information Systems 39(1): 22-44. Disponível em http://dx.doi.org/ $10.1016 / j$.is.2013.07.002 (Accessado 2 Setembro 2017).
Esposito, C., Cotroneo, D. and Russo, S. (2013). On reliability in publish/subscribe services, Computer Networks 57(5): 1318-1343. Disponível em http://dx.doi.org/10.1016/j.comnet.2012.10.023 (Accessado 2 Setembro 2017).

Eugster, P. T., Felber, P. A., Guerraoui, R. and Kermarrec, A.-M. (2003). The many faces of publish/subscribe, ACM computing surveys (CSUR) 35(2): 114-131.

Fernández-Sáez, A. M., Genero, M. and Chaudron, M. R. V. (2013). Empirical studies concerning the maintenance of uml diagrams and their use in the maintenance of code: A systematic mapping study, Information and Software Technology 55(7): 11191142. Disponível em http://dx.doi.org/10.1016/j. infsof.2012.12.006 (Accessado 2 Setembro 2017).

ISO:12052, N. P. . (2016). Digital imaging and communications in medicine (dicom) standard. Disponível em http://medical.nema.org/ (Acessado 18 Junho 2017).

Kaur, M. and Wasson, V. (2015). Roi based medical image compression for telemedicine application, Procedia Computer Science 70: 579-585.

Maani, R., Camorlinga, S. and Arnason, N. (2012). A parallel method to improve medical image transmission, Journal of Digital Imaging 25(1): 101109.

Maldonado, J. M. S. d. V., Marques, A. B. and Cruz, A. (2016). Telemedicine: challenges to dissemination in brazil, Cadernos de saude publica 32.

Mell, P. and Grance, T. (2011). The nist definition of cloud computing, National Institute of Standards and Technology, Information Technology Laboratory .

Mildenberger, P., Eichelberg, M. and Martin, E. (2002). Introduction to the dicom standard, European Radiology 12(4): 920-927.

Ojog, I. and Arias-Estrada, M. (2013). A cloud scalable platform for dicom image analysis as a tool for remote medical support, eTELEMED 2013, The ... (c): 246-249.

Ootsu, K., Yokota, T. and Ohkawa, T. (2016). A consideration on compression level control for dynamic compressed data transfer method, Computational Science and Computational Intelligence (CSCI), 2016 International Conference on, IEEE, pp. 637-640.

Pianykh, O. S. (2008). Digital imaging and communications in medicine (DICOM): a practical introduction and survival guide, Springer Science \& Business Media.

Puthal, D., Sahoo, B. P. S., Mishra, S. and Swain, S. (2015). Cloud computing features, issues, and challenges: A big picture, Proceedings - 1st International Conference on Computational Intelligence and Networks, CINE 2015 pp. 116-123.

Rallapalli, S., Gondkar, R. and Ketavarapu, U. P. K. (2016). Impact of processing and analyzing healthcare big data on cloud computing environment by implementing hadoop cluster, Procedia Computer Science 85: 16-22. 
Righi, R. D. R. (2013). Elasticidade em cloud computing: conceito, estado da arte e novos desafios, Revista Brasileira de Computação Aplicada 5(2): 2-17. Disponível em http://www.upf.br/ seer/index.php/rbca/article/view/3084 (Accessado 2 Setembro 2017).

Righi, R., Rodrigues, V. F., Da Costa, C. A., Galante, G., De Bona, L. C. E. and Ferreto, T. (2016). Autoelastic: Automatic resource elasticity for high performance applications in the cloud, IEEE Transactions on Cloud Computing 4(1): 6-19.

Tarkoma, S. (2012). Publish/subscribe systems: design and principles, John Wiley \& Sons.

Teng, C. C., Mitchell, J., Walker, C., Swan, A., Davila, C., Howard, D. and Needham, T. (2010). A medical image archive solution in the cloud, Proceedings 2010 IEEE International Conference on Software Engineering and Service Sciences, ICSESS 2010, pp. 431-434.

Teranishi, Y., Kawakami, T., Ishi, Y. and Yoshihisa, T. (2017). A large-scale data collection scheme for distributed topic-based pub/sub, Computing, Networking and Communications (ICNC), 2017 International Conference on, IEEE, pp. 230-236.

Varia, J. and Mathew, S. (2014). Overview of amazon web services, Amazon Web Services .

Westin, M., Melin, J., Nordgren, А. and Eriksson, J. (2015). Systems and devices for encrypting, converting and interacting with medical images using a mobile device. Disponível em https:// www.google.com/patents/US20150278444 (Accessado 15 Março 2017).

Westin, M., Melin, J., Nordgren, A., Eriksson, J. and Thurman, A. (2016). Systems and devices for encrypting, converting and interacting with medical images. Disponível em https: //www. google. com/patents/US20160037057 (Acessado 20 Março 2017).

WHO (1998). A health telematics policy. Disponível em http://www.who.int/iris/handle/10665/63857 (Acessado 20 Junho 2017). 